\title{
L'action publique en Afrique, entre normes pratiques, dynamiques politiques et influences externes
}

Introduction

Philippe Lavigne Delville et Sylvie Ayimpam

\section{OpenEdition \\ Journals}

Édition électronique

URL : http://journals.openedition.org/anthropodev/660

DOI : 10.4000/anthropodev.660

ISSN : 2553-1719

Éditeur

APAD - Association pour l'anthropologie du changement social et du développement

Édition imprimée

Date de publication : 1 décembre 2018

Pagination : 25-41

ISBN : 979-10-93476-06-3

ISSN : 2276-2019

\section{Référence électronique}

Philippe Lavigne Delville et Sylvie Ayimpam, «L'action publique en Afrique, entre normes pratiques, dynamiques politiques et influences externes », Anthropologie \& développement [En ligne], 48-49 | 2018, mis en ligne le 18 juillet 2019, consulté le 24 septembre 2020. URL : http://journals.openedition.org/ anthropodev/660; DOI : https://doi.org/10.4000/anthropodev.660

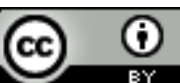

La revue Anthropologie \& développement est mise à disposition selon les termes de la Licence Creative Commons Attribution 4.0 International. 


\section{L'action publique en Afrique, entre normes pratiques, dynamiques politiques et influences externes}

Introduction

Philippe Lavigne Delville et Sylvie Ayimpam

Un champ de recherche dynamique

L'État en action en Afrique, et plus largement l'action publique, constituent un champ de recherche dynamique. Trois ouvrages collectifs ou dossiers de revue y ont été spécifiquement consacrés, au cours de ces dernières années. Le présent dossier s'inscrit donc dans une riche production récente, marquée également par plusieurs autres ouvrages (Ridde et Jacob, 2013 ; Bierschenk et Olivier de Sardan, 2014 ; Olivier de Sardan et Ridde, 2014 ; Olivier de Sardan et Piccoli, 2018) et numéros de revue sur des thèmes connexes (Chauveau, 2017 ; Petiteville, 2017).

Géographes, démographes, économistes, politistes, ont participé à l'ouvrage intitulé L'État réhabilité en Afrique (Grégoire, Kobiané et Lange, 2018), qui propose un large panorama sur les politiques sectorielles en Afrique, avec un regard qui veille à inscrire ces politiques dans leur contexte social et politique et dans leur histoire. Dans leur chapitre d'état des lieux, Emmanuel Grégoire et Marie-France Lange (2018) retracent l'évolution des politiques publiques, en particulier économiques, depuis les Indépendances, mettant en perspective la situation spécifique des États africains contemporains, qui sont à la fois réhabilités dans les discours internationaux mais toujours fortement contraints dans leur autonomie. Pour ces auteurs, attentifs à ne pas surestimer le rôle de l'aide :

"[...] c'est autant l'impact de la mondialisation que celui de la dépendance et des réactions à celle-ci qu'il faut étudier pour comprendre les nouvelles conditions de l'action publique » (Grégoire et Lange, $2018: 21$ ). 
Les recompositions du rôle de l'État, la multiplication des acteurs, les références croissantes au New Public Management, sont en effet des tendances mondiales, qui ne sont pas spécifiques aux " pays sous régime d'aide " même si elles y prennent des formes particulières (Fresia et Lavigne Delville, 2018). Une telle approche historique est indispensable, et met en perspective les dynamiques contemporaines: la multiplicité des acteurs internationaux est le produit des ajustements structurels, et non pas quelque chose de naturel, elle pose de nombreuses questions de légitimité. Mais s'il est clair que la déclaration de Paris, signée en 2005, affirme remettre l'État et les politiques au centre, que peut-on dire de la réalité de cette " réhabilitation » ?

Peu de temps auparavant, les revues Anthropologie \& développement et Gouvernement et action publique avaient chacune proposé un état des lieux sur la question de l'action publique et des politiques publiques en Afrique, respectivement du point de vue de la socio-anthropologie du développement et de la science politique. Ces deux disciplines ont des questionnements très proches. En interrogeant la façon dont des acteurs variés, nationaux et internationaux, étatiques ou non, s'emparent de problèmes, les définissent comme publics et agissent pour y répondre, il $s^{\prime}$ agit dans les deux cas de mettre en lumière les formes spécifiques que prend l'action publique dans les pays africains, porteurs de dynamiques sociales et politiques originales, où l'aide est fortement présente dans de nombreux secteurs, pour le meilleur ou pour le pire. Et finalement de mieux comprendre à la fois l'État en action et le rôle que jouent les différents groupes sociaux et les acteurs internationaux. Des deux côtés, il s'agit d'étudier les rapports entre acteurs variés dans la définition et la mise en place de réponses à des problèmes publics ou collectifs, en prenant au sérieux les liens entre policies (les politiques publiques), politics (les enjeux de pouvoir, la compétition électorale) et polities (les régimes politiques, les débats de choix de société), la relativement faible institutionnalisation des institutions - laquelle résulte de leur sensibilité aux influences politiques et de la forte personnalisation des relations avec les usagers - et enfin les logiques d'extraversion (Bayart, 1999). Dès lors qu'ils s'intéressent à l'action publique, chercheurs en socio-anthropologie du développement et en science politique se donnent donc des objets voisins, qu'ils abordent en fonction de leurs références, de leurs cadres 
théoriques, de leur rapport au terrain, avec des emprunts réciproques mais que l'on peut juger encore trop timides.

Les questionnements ne sont cependant pas exactement les mêmes. En s'appuyant sur les acquis des recherches sur les interventions de développement, les relations entre usagers et agents des services publics ou collectifs, les réformes des administrations, ou les transferts de modèle, il s'agit pour la socio-anthropologie d'élargir son questionnement, d'une part en remontant en amont des interventions et des dispositifs de terrain vers les lieux de production des normes, des modèles, des politiques, et d'autre part en déplaçant le regard vers des lieux ou des espaces qui se situent en dehors ou en marge des thèmes d'intervention de l'État et de I'aide (Lavigne Delville, 2016). À travers cela, il s'agit aussi de prendre en compte la grande diversité des configurations d'action publique et des formes qu'y prend l'aide, et de banaliser le "développement » en le considérant comme une forme spécifique d'action publique, en partie extravertie et internationalisée, qui a été historiquement mise en place dans les pays "sous régime d'aide". Du point de vue de la science politique, il s'agit de :

"[...] participer aux débats sur la construction de l'État à travers l'étude de la configuration des politiques publiques sur le continent en les appréhendant comme des expressions et des outils de la fabrication de l'État, [ce qui suppose que] l'analyse des secteurs de politiques publiques en Afrique soit systématiquement mise en articulation avec la sociologie de l'action publique et la sociologie historique de l'État » (Darbon et Provini, 2018 : 11).

$\mathrm{Si}$ « les débats sur l'État et le pouvoir africains ont été posés en premier lieu par les anthropologues" (Gazibo et Thiriot, 2009: 22) étudiant le fonctionnement quotidien de l'État local ou décentralisé, ces deux champs scientifiques convergent pour poser l'action publique comme un objet de recherche permettant de renouveler le regard sur l'État en Afrique.

Au-delà des différences de sensibilité ou d'approche, et parfois de rapport au terrain, ces différents travaux appellent en tout cas à prendre au sérieux l'enchâssement de l'action publique et des politiques publiques 
dans l'histoire, la société, le politique. Le présent dossier poursuit la valorisation scientifique du $12^{\mathrm{e}}$ colloque international de I'APAD $^{1}$, qui avait pour thème " La fabrique de l'action publique dans les pays 'sous régime d'aide' ", et se situe dans la continuité du $n^{\circ} 45$ d'Anthropologie \& développement, lui aussi issu de ce colloque. Tout en explorant des formes variées de coproduction de l'action publique par différents acteurs, ce premier dossier thématique proposait un cadre d'analyse et mettait l'accent sur le caractère internationalisé et extraverti de l'action publique en Afrique. Les textes réunis dans le présent dossier ${ }^{2}$ poursuivent cette exploration de facettes variées de l'action publique en Afrique, des pratiques locales aux rapports entre bailleurs de fonds et gouvernements nationaux. Certaines contributions portent sur des secteurs particuliers d'activités: la santé, avec les médicaments mis à disposition contre le paludisme au Bénin et au Ghana (Pourraz, Baxerres et Cassier); l'éducation, avec la fabrique des statistiques scolaires au Bénin (Fichtner) ; l'électricité, avec les formes de "privatisation " informelle de l'électricité en république démocratique du Congo (Mpiana Tshitenge); le foncier, avec la réforme de la gestion foncière visant l'instauration du cadastre au Mali (Bertrand). Tandis que d'autres explorent des perspectives variées de la fabrique de l'action publique, telles les politiques de "bonne gouvernance » au Burundi (Hirschy), la gouvernance de la laïcité au Burkina Faso (Ouédraogo), ou encore les concurrences entre services techniques et ONG (Kaboré). Les articles insistent sur la pluralité des acteurs - tant nationaux qu'internationaux - engagés dans l'action publique, questionnent le pouvoir d'influence des acteurs internationaux, mettent en avant des enjeux de contrat social, et finalement nous appellent à prendre au sérieux l'enchâssement social et politique de l'action publique, et son inscription dans des contextes, des histoires, des réseaux d'acteurs. Trois grands axes sont particulièrement mis en avant dans ces articles.

1 "La fabrique de l'action publique dans les pays 'sous régime d'aide' ", colloque coorganisé par I'APAD, l'université d'Abomey-Calavi, le LADyD, le LASDEL et I'IRD, Cotonou, 17-20 novembre 2015.

2 Dont deux ont bénéficié de l'atelier d'écriture organisé, suite au colloque de Cotonou, par I'APAD et le LADyD en septembre 2016 à Cotonou. L'article d'Issifou Abou Moumouni, publié dans le $n^{\circ} 45$, avait aussi bénéficié de cet atelier. 
La gouvernance par le bas : carences institutionnelles, concurrences entre acteurs et normes pratiques

Trois textes de ce dossier explorent la question des rapports entre acteurs publics et privés dans l'action publique, des relations de concurrences et de complémentarités qu'ils entretiennent, entre eux et avec les administrations publiques, et de leurs pratiques effectives, au-delà des normes.

On le sait, tant au Nord qu'au Sud, le tournant néolibéral met en cause la centralité de l'État et contribue à une " démographie galopante des acteurs pertinents dans les politiques publiques " (Massardier, $2003: 76$ ), tantôt perçue comme signe de complémentarité et d'efficience (la participation d'acteurs associatifs ou privés d'un côté, des usagers de l'autre, étant censée permettre une plus grande pertinence des décisions publiques et une plus grande efficacité de la mise en œuvre), tantôt comme porteuse d'instabilité, d'incohérences, d'inefficacité, voire d'ingouvernabilité (ibid. : 140). Cette tendance est encore plus marquée en Afrique avec la multiplication, depuis les années 1990, des ONG, projets de développement, associations, collectivités locales, fondations, mécènes, mouvements religieux, etc., tant nationaux qu'internationaux. La dynamique des champs organisationnels, les rapports entre organisations, et les relations de complémentarité, concurrence, superposition, évitement, neutralisation, etc. qu'elles entretiennent entre elles, sont une clé d'analyse pour comprendre la gouvernance des services publics et collectifs.

Une seconde clé, complémentaire, porte sur l'analyse des pratiques. II s'agit de mettre en évidence les régularités, les routines qui émergent des interactions répétées entre acteurs (usagers comme fournisseurs de services) dans ce contexte de multiplication des acteurs intervenant sur les problèmes publics. C'est cela même la définition de la gouvernance, au sens empirique et non normatif du terme qui est celui de la socioanthropologie (Blundo et Le Meur, 2009). Entre pluralité des normes, carences institutionnelles, décalages d'intérêts et stratégies opportunistes, les pratiques sont en effet structurellement, même si c'est à des degrés variables, en décalage par rapport aux normes censées régir un secteur donné. L'analyse "par le bas » est seule à même de décrire la réalité des 
pratiques et donc le fonctionnement réel des services et des administrations, les décalages entre les normes et les pratiques étant une clé d'entrée particulièrement riche pour interroger les représentations et les pratiques des acteurs de l'action publique, usagers comme " offreurs de service ", et à partir de là de mettre au jour les normes pratiques qui les régissent, c'est-à-dire :

"[...] les diverses régulations informelles, tacites ou latentes, qui sous-tendent les pratiques des acteurs, non conformes aux normes officielles ou formelles, et qui expliquent par leur existence même la convergence relative de ces pratiques" (Olivier de Sardan, 2010: 12).

Ce qui permet ensuite d'identifier les différentes raisons de ces décalages, les formes de bricolages et d'innovations que les uns et les autres mettent en place, pour tenter de stabiliser des dispositifs, en améliorer l'effectivité et/ou en tirer partie, dans des stratégies qui peuvent être adaptatives (qui " permettent de rendre des normes abstraites et impersonnelles compatibles avec des contextes particuliers et des situations singulières"), transgressives (qui "nuisent gravement à la délivrance de services publics de qualité ou au fonctionnement efficient des administrations ») ou palliatives (qui «facilitent de façon "bricolée" la délivrance d'un service, tout en ouvrant la porte à des privatisations informelles ») (Olivier de Sardan, $2014:$ 10-11).

La place des référentiels internationaux et les modalités par lesquelles ils peuvent être importés dans l'espace national sont particulièrement bien illustrées par l'exemple de l'arène scolaire au Bénin. Peut-on avoir une même vision de l'école, peut-on la gouverner de la même manière, selon qu'on est guidé par une politique internationale de l'éducation pour tous ou par une politique nationale? S'intéressant à la production des statistiques scolaires au Bénin, Sarah Fichtner souligne comment l'objectif de l'Éducation pour tous, centré autour des mots clés du partenariat éducatif, du volontarisme et de l'efficacité, implique un changement de la politique éducative, en particulier en ce qui concerne les effectifs scolaires et le taux de redoublement, et comment les statistiques scolaires, censées objectiver la situation des écoles et déterminer les décisions de l'administration scolaire, sont soumises aux logiques d'intérêts des directeurs d'école : 
"La production des chiffres, n'est pas seulement une tâche d̀ accomplir pour informer l'État, les ONG et les partenaires techniques et financiers, elle est un outil de la gouvernance locale dans un contexte globalisé : elle touche aux relations entre directeur et parents d'élèves, au financement de l'école, à la dotation en manuels scolaires, aux vivres de la cantine scolaire et à la sélection scolaire à travers les notes et les pratiques du redoublement " (Fichtner, ce numéro : 46).

Les directeurs d'école font face à des injonctions contradictoires (limiter le redoublement et avoir de bons taux de réussite aux examens), à des dotations budgétaires insuffisantes, à des pressions des associations de parents d'élèves. Ils ont aussi leurs propres stratégies de carrière, et avoir réussi à obtenir l'ouverture de nouvelles classes est un atout. Dès lors :

"La fabrique des chiffres devient un "jonglage" pour présenter une image réaliste et harmonieuse du développement progressif de l'école et de sa "bonne gouvernance", en même temps que pour atteindre les différents objectifs (financiers, carriéristes) des acteurs engagés dans leur production. Le contrôle par les chiffres de la gestion scolaire, de son évolution et de sa "situation morale", instauré pour gérer la distance et la méfiance du gouvernement, est alors limité par le caractère instrumental de ces chiffres dans l'arène scolaire et l'insuffisance des moyens pour les vérifier » (ibid. : 54).

La manipulation des statistiques relève ici plutôt de stratégies adaptatives, face aux injonctions contradictoires auxquelles sont soumis les directeurs d'école. Cette contribution montre comment l'exigence implicite des bons chiffres par les politiques internationales de l'aide, conduit à des petits arrangements par les acteurs à différents niveaux, favorisant le travestissement de la réalité et la fabrication de plusieurs réalités de papier, légitimant les objectifs différents des acteurs impliqués dans l'arène scolaire.

L'ampleur des arrangements avec la norme est sans commune mesure dans les services de base qui font face à des carences institutionnelles fortes. Jean-Pierre Mpiana Tshitenge nous fait plonger dans le quotidien du service de l'électricité à Kisenso, dans la périphérie de Kinshasa. Face aux multiples défaillances de la Société nationale d'électricité (SNEL) et de ses agents, les usagers ont développé des modes palliatifs d'accès à 
l'électricité. Les agents de la SNEL acceptent des raccordements illégaux, dont certains sont ensuite régularisés et d'autres non. Les usagers se cotisent parfois pour financer un transformateur ou une prolongation de réseau. Ils accordent à d'autres l'accès à leur compteur, à travers un réseau dense et indémêlable de fils électriques qui traversent les rues. Tirant des profits individuels de ces arrangements, les agents de la SNEL sont des parties prenantes actives de cette privatisation informelle de la distribution d'électricité, qui contribue à étendre l'accès au service au-delà de l'offre officielle tout en aggravant la crise financière de la compagnie.

"Les usagers, plus complices que victimes, semblent préférer cette forme de régulation très pratique qui assouplit en coût et en temps les procédures formelles d'accès à l'électricité. Ainsi, ces modes alternatifs de délivrance et d'accès à l'électricité font émerger des espaces de régulation conjointe alliant la régulation de contrôle de l'entreprise étatique et la régulation autonome des acteurs locaux » (Mpiana Tshitenge, ce numéro : 68).

Laissant libre cours aux arrangements informels entre acteurs locaux et entre agents du service publics et acteurs locaux, ce processus permet à l'État de conserver en apparence ses prérogatives et ses droits souverains, tout en choisissant délibérément de ne pas les exercer complètement afin d'obtenir le consentement des citoyens. Cette forme de délivrance palliative du service public d'électricité instaure un espace de régulation conjointe où s'articulent régulations de contrôle et régulations autonomes, dans des arrangements " par le bas " qui rappellent à certains égards les partenariats publics-privés prônés par le référentiel néolibéral.

À travers le cas de l'Union fraternelle des croyants (UFC), à Dori (Burkina Faso), Koudbi Kaboré étudie quant à lui les concurrences institutionnelles, par l'analyse de l'évolution des relations entre services techniques agricoles, ONG et administration territoriale dans le champ du développement rural, et met en évidence les rivalités sourdes qui se développent entre ces acteurs de la sécurité alimentaire pour la supervision et le contrôle des organisations paysannes. Fondée en 1969, I'UFC a la particularité d'être une ONG confessionnelle, promouvant le dialogue interreligieux entre catholiques et musulmans en même temps que le développement agricole, avec une gouvernance partagée entre représentants de ces deux religions. Bénéficiant de soutiens des réseaux 
de l'Église, elle s'est rapidement imposée comme un acteur central de l'aide alimentaire, puis du développement agricole dans la région, par l'appui à des groupements maraîchers, et des aménagements hydroagricoles. L'ONG s'est progressivement structurée, suivant l'évolution des politiques nationales qui veulent faire des ONG des auxiliaires de l'État, avant de connaître une grave crise de gouvernance qui a induit une mise en sommeil. Grâce à la reprise de ses financements internationaux, I'UFC se relance en 1999, à travers une restructuration et une professionnalisation de son organisation, au moment où l'État réorganise sa politique et son emprise sur la structuration du monde rural : les groupements doivent se structurer au sein de la chambre d'agriculture, les services techniques doivent s'autofinancer par des prestations de service. Alors que I'UFC reste présentée comme un exemple par l'administration territoriale, les relations se tendent avec les services techniques qui lui reprochent de conserver la tutelle et le monopole de l'appui aux groupements qu'elle a créés, et d'avoir ses propres équipes techniques au lieu de mobiliser (moyennant finance) les services de l'État. Les agents des services techniques de l'agriculture contestent la bienveillance de l'administration territoriale envers I'UFC, renforcée avec la crainte de la radicalisation religieuse, et qui lui permet de développer des pratiques hors normes et de se soustraire à leur contrôle. Les tensions qui opposent les acteurs de I'UFC aux structures étatiques apparaissent ainsi avant tout comme le reflet des évolutions affectant les relations entre les ONG et les structures étatiques depuis le retour de l'État sur le devant de la scène du développement au début des années 2000 :

"Faute de ressources publiques structurelles suffisantes, [les services techniques de l'État] sont eux-mêmes amenés à vendre leurs services. Devenus eux-mêmes des courtiers locaux, ils ne peuvent qu'entrer en concurrence avec les acteurs privés sur le terrain du développement » (Kaboré, ce numéro : 116).

Et cela suscite rivalités et jalousies. 
Politique, enjeux de contrat social et formulation des problèmes publics

Les tensions entre acteurs de l'action publique ne sont pas seulement le reflet de concurrences institutionnelles ou d'enjeux de rente financière. Elles peuvent aussi traduire des divergences entre projets de société, des luttes pour faire émerger des problèmes publics ou pour imposer une façon de les penser. L'action publique est aussi une question de "polity ", de façon de penser la société, de contrat social (Leca, 2012). Deux textes illustrent cette dimension.

La question de l'équilibre entre religions au Burkina Faso est un sujet sensible. La contribution de Yacouba Ouédraogo analyse le processus ayant conduit à la mise à l'agenda de la gouvernance de la laïcité au Burkina Faso dans un contexte de réislamisation en Afrique subsaharienne.

"Au Burkina Faso, les associations musulmanes francophones, insatisfaites de la gestion de la laïcité par l'État, engagent une lutte à partir du début des années 1990, non pas pour remettre en cause la laïcité constitutionnelle, mais pour réclamer plus d'équité dans le traitement des confessions religieuses " (Ouédraogo, ce numéro: 119).

Le retour de la démocratie en 1990 a, comme dans d'autres pays, ouvert un espace pour les revendications religieuses. Récentes, les jeunes organisations de lettrés francophones musulmans contestent la place historique du catholicisme dans l'État et les déséquilibres entre religions dans les jours fériés, la présence à la télévision, les postes de représentation dans les instances de dialogue politique. Elles critiquent la politique de l'État vis-à-vis du voile islamique. Ces contestations sont mises sur la scène publique à l'occasion d'un colloque initié par une association musulmane en 2007. En 2011, la crise sociopolitique qu'a vécue le régime de Blaise Compaoré a amené ce dernier à créer un Conseil consultatif sur les réformes politiques, qui intègre les organisations religieuses. Les associations musulmanes revendiquent la création d'un observatoire de la laïcité. L'État refuse mais organise en 2012 un Forum national sur la laïcité, qui suscitera de vifs débats sur le sens de la laïcité au Burkina Faso, entre une vision positive des religions et les craintes d'une trop forte présence 
dans l'État, et des compromis sur les questions de représentation et de présence dans l'espace médiatique.

Cette frange spécifique des organisations musulmanes illustre ainsi "l'émergence d'une société civile musulmane qui, sans être contestatrice, frondeuse ou rebelle, s'inscrit dans un courant critique des rapports entre l'État et les confessions religieuses " (Ouédraogo, ce numéro : 137-138), et se revendique de la laïcité pour poser le problème des rapports entre religions dans leur traitement par l'État et dans l'espace public, et tenter de les renégocier. Face au spectre de l'islamisme qui se développe dans le monde et également dans la sous-région, ce débat sur la laïcité représente finalement aussi une opportunité pour les différents protagonistes, l'État en premier lieu : au-delà de la revendication d'un traitement égalitaire par les associations musulmanes, il s'agissait aussi pour l'État de contrer les risques de montée du djihadisme et pour les catholiques et les protestants de mettre en avant leur crainte d'une remise en cause de leur liberté religieuse. Ce cas montre comment la revendication d'un groupe d'acteurs a été prise en compte dans un contexte politique spécifique, et comment leur façon de poser le problème a été reformulée en fonction d'enjeux locaux et globaux.

C'est d'un autre type de problème public, le foncier, que traite l'article de Monique Bertrand. Suivant le double fil des politics et des policies autour d'une question qui est au cœur du contrat social, l'auteure propose une lecture particulièrement fine de la façon dont l'urgence d'une réforme foncière est - une nouvelle fois - mise à l'agenda par le gouvernement malien. Au lendemain des élections législatives de 2014 censées tourner la page de la crise politique de 2012 (coup militaire et occupation djihadiste), cette réforme s'insère en effet " dans un contexte présenté comme de 'sortie de crise' : non pas dans un cadre routinier de renforcement de capacités institutionnelles [...], mais en opération de sauvetage de la cohésion sociale et du crédit territorial de l'État " (Bertrand, ce numéro: 143-144). Les multiples conflits fonciers et spoliations ne sont pas nouveaux, mais ils ont été particulièrement médiatisés à cette époque. Ils vont être mis en avant par le pouvoir dans une opération de critique de la gouvernance précédente et de mise en scène d'une volonté d'assainir le secteur, preuves du volontarisme du nouveau pouvoir. L'auteure suit pas à pas cette nouvelle tentative de réforme, depuis sa mise sur agenda, en 
interrogeant les cadrages contradictoires dont elle est l'objet et l'élaboration des stratégies d'action, puis en examinant les enjeux de captation et d'instrumentalisation politique de la rente foncière, d'arbitrage des concurrences à l'œuvre autour de différentes compétences administratives et des groupes sociaux qui en usent. Au départ, le discours politique dénonce vigoureusement les pratiques frauduleuses dont sont victimes les simples citoyens, et met en avant des mesures d'urgence. Mais il glisse vite sur la mise en avant - une nouvelle fois - d'une solution miracle mais peu définie, le cadastre, dont les liens avec le problème politique identifié ne vont pas de soi.

La formulation du problème comme problème de gouvernance, posant la question des rapports entre État et citoyens, se transforme en formulation technicisée et bureaucratisée. L'apparente volonté réformatrice se heurte rapidement aux corporatismes, aux rivalités institutionnelles entre ministères. La ressource foncière est un énorme enjeu de rente et suscite de constantes tensions entre des bureaucraties jalouses de leur information, peu disposées à la concertation, promptes à déclarer les autres incompétentes pour justifier leurs propres raccourcis de procédure. Trois ministres se succèdent, aux styles personnels très différents. Le budget estimé dépasse largement les moyens de l'État malien, qui réduit ses engagements, et les bailleurs de fonds ne suivent pas. Sur le terrain, " la suspension décrétée d'en haut [de la délivrance de titres] n'a pas d'effet autre que pervers, et montre la force d'arrangement de la demande avec la bureaucratie locale " (Bertrand, ce numéro : 160). Cruciale, mais verrouillée par un enchevêtrement complexe d'institutions, d'intérêts et de routines administratives, la volonté de traiter la question foncière se confronte à la difficulté de l'action politique, à laquelle se couple la dépendance aux moyens externes.

Une coproduction internationalisée de l'action publique : résistances et impositions dans les relations d'aide

Les deux derniers textes de ce dossier abordent de front la question des rapports complexes entre institutions nationales et internationales, et celle de la volonté et de la capacité des acteurs et des institutions nationales à faire valoir leurs priorités et leurs stratégies, dans un contexte marqué par 
la dépendance aux financements externes et la prévalence des modèles imposés.

En examinant les dispositifs d'approvisionnement en médicaments contre le paludisme au Bénin et au Ghana, Jessica Pourraz, Carine Baxerres et Maurice Cassier discutent des rapports entre acteurs transnationaux et nationaux dans ce domaine spécifique de l'action publique, pris en charge par un oligopole d'organisations transnationales centralisées. Comme plusieurs pays africains, le Ghana et le Bénin dépendent des subventions du Fonds mondial de lutte contre le sida, la tuberculose et le paludisme et de la President's Malaria Initiative des États-Unis pour approvisionner en médicaments antipaludiques leurs secteurs publics, voire privés. Pour des raisons d'économies d'échelle, ces organisations ont, à partir du milieu des années 2000, progressivement pris en charge les commandes de médicaments, générant de nouveaux réseaux d'approvisionnement qui entrent en concurrence avec les dispositifs nationaux existants.

"La multiplication des réseaux et des circuits d'achat génère une fragmentation du système d'approvisionnement dans les pays qui conduit à des dysfonctionnements dans la gestion des médicaments tels que des ruptures ou des excédents d'approvisionnement " (Pourraz, Baxerres et Cassier, ce numéro : 180).

Les acteurs transnationaux ont alors cherché à impulser certaines formes d'appropriation au niveau des États afin de répondre aux problèmes d'inefficacité des systèmes d'approvisionnement qu'ils ont euxmêmes générés. Le cas de la mise à disposition des médicaments contre le paludisme dans ces deux pays illustre bien le fait que les dispositifs d'action publique sont issus de processus de négociations pluri-acteurs (État, bailleurs, institutions locales, opérateurs), et que ces négociations tendent aussi à se poursuivre lors de la mise en œuvre, notamment du fait des réactions des populations et des institutions locales. La situation des deux pays est cependant différente, en particulier du fait de l'existence au Ghana de firmes pharmaceutiques. Mais, en conditionnant les aides financières à l'achat de médicaments pré-qualifiés par l'Organisation mondiale de la santé, ces acteurs transnationaux excluent les firmes ghanéennes de leurs appels d'offres (qui bénéficient avant tout aux firmes américaines), ce qui est contesté par d'autres acteurs du développement, qui prônent au contraire d'appuyer la montée en gamme des industries 
nationales. Les dispositifs nationaux de distribution demeurent très spécifiques à ces deux pays, du fait de l'histoire du secteur, mais l'organisation du secteur doit autant, sinon plus, à la façon dont les acteurs transnationaux adaptent leurs stratégies dans un contexte donné qu'à une réelle capacité nationale de formulation des politiques.

"Ce paradoxe, relevé aussi bien au Bénin qu'au Ghana, découle de la contradiction structurelle de l'aide internationale au développement qui oscille entre le principe d'une plus grande autonomie des institutions nationales, prônée par l'objectif de l'appropriation, et celui d'une plus grande efficacité de sa mise en œuvre en réponse aux logiques internes des institutions internationales. Ces éléments démontrent l'usage stratégique et les limites fondamentales du concept d'appropriation" (Pourraz, Baxerres et Cassier, ce numéro : 187-188).

Dans ce cas, le contrôle des moyens financiers donne aux organisations transnationales un pouvoir central sur la filière. II ne suffit pas toujours, comme le montre l'article de Justine Hirschy, qui interroge les politiques de "bonne gouvernance» au Burundi. Elle questionne les relations entre l'État burundais (élites au pouvoir et administration) et les bailleurs de fonds (principalement la Belgique et la Banque mondiale) autour de la stratégie nationale de bonne gouvernance. Élu en 2000 à la suite de la transition post-guerre civile, le président Pierre Nkurunziza avait fait de la bonne gouvernance un slogan central. Il a créé un ministère dédié, confié à l'opposition, et lancé la définition d'une stratégie nationale. Les financements affluent. Plus encore, l'élite du parti au pouvoir n'a de cesse d'élaborer des mesures visant à neutraliser son potentiel de changement. Définie tardivement, la stratégie s'aligne formellement sur les priorités définies par les bailleurs de fonds, mais manque de contenu, malgré les pressions de ces derniers.

"Alors que le gouvernement s'était largement aligné au niveau discursif sur les standards internationaux en termes de "bonne gouvernance", les pratiques semblent peu à peu s'en éloigner. À mesure que les élections de 2015 se rapprochent, l'élite du CNDDFDD au pouvoir va non seulement réinterpréter les normes internationales, mais également les détourner et les bloquer afin d'assurer la survie politique du régime » (Hirschy, ce numéro : 195). 
Restrictions à la liberté d'expression et arrestations arbitraires recommencent. L'enjeu du maintien au pouvoir est tel que le régime préfère perdre des soutiens financiers. L'influence exercée par les bailleurs de fonds se situe avant tout au niveau bureaucratique, c'est-à-dire par des interactions entre les fonctionnaires de l'administration burundaise et internationale ainsi qu'au niveau des discours politiques. Mais, les questions de "bonne gouvernance" sont hautement politiques et les tentatives des bailleurs de fonds pour influencer les pratiques dans ce domaine sont mises en échec lorsqu'elles se heurtent aux enjeux de maintien au pouvoir.

"Élément central du processus de paix, en 2000, les politiques de "bonne gouvernance", n'ont pas atteint les objectifs escomptés et c'est sous le signe de la violence ainsi que de la peur que débute le troisième mandat du président Nkurunziza. En parallèle, les récentes coupes des bailleurs de fonds ont, en réduisant les capacités de redistribution du régime, non seulement conduit à un rétrécissement des cercles bénéficiaires, mais également renforcé les besoins du CNDD-FDD d'asseoir son pouvoir par la coercition " (Hirschy, ce numéro : 212).

Ces deux textes mettent bien en exergue l'ambivalence des rapports entre acteurs nationaux et acteurs internationaux de l'action publique autour de l'aide internationale, ainsi que l'équivocité des processus par lesquels cette action peut être négociée, adaptée, contestée, voire détournée. Loin des schémas manichéens opposant acteurs nationaux et internationaux, ils donnent à voir l'enchevêtrement des acteurs, mais dans des configurations très différentes, où la capacité des acteurs nationaux varie considérablement.

Balayant une large gamme de configurations, depuis l'émergence des problèmes publics jusqu'aux contradictions de la mise en œuvre, les articles réunis dans ce dossier contribuent à affiner notre compréhension des dilemmes et des enjeux de l'action publique en Afrique, ainsi que leur enchâssement social et politique, et leur inscription dans la globalisation. 


\section{Bibliographie}

BAYART J.-F., 1999, "L'Afrique dans le monde: une histoire d'extraversion ", Critique internationale, 5(1) : 97-120.

BIERSCHENK T. et OLIVIER DE SARDAN J.-P. (éds), 2014, States at Work. Dynamics of African Bureaucracies, Coll. Africa-Europe Group for Interdisciplinary Studies, vol. 12, Leiden, Brill.

BLUNDO G. et LE MEUR P.-Y. (éds), 2009, The Governance of Daily Life in Africa: Ethnographic Explorations of Public and Collective Services, vol.19, Leiden, Brill Academic Pub.

CHAUVEAU J.-P. (éd.), 2017, " La gouvernementalité rurale dans les pays du Sud ", Critique internationale, 75, dossier, Paris, Presses de sciences po.

DARBON D. et PROVINI O., 2018, " "Penser l'action publique" en contextes africains. Les enjeux d'une décentration ", Gouvernement et action publique, 2(2) : 9-29.

FRESIA M. et LAVIGNE DELVILLE P. (éds), 2018, Au coeur des mondes de l'aide internationale. Regards et postures ethnographiques, Paris/Marseille/ Montpellier, Karthala/IRD/APAD.

GAZIBO M. et THIRIOT C. (éds), 2009, Le politique en Afrique. État des débats et pistes de recherche, Paris, Karthala.

GRÉGOIRE E., KOBIANÉ J.-F. et LANGE M.-F. (éds), 2018, L'État réhabilité en Afrique. Réinventer les politiques publiques à l'ère néolibérale, Paris, Karthala.

GRÉGOIRE E. et LANGE M.-F., 2018, "Politiques publiques, développement et mondialisation en Afrique subsaharienne ", in GRÉGOIRE E., KOBIANÉ J.-F. et LANGE M.-F. (éds), L'État réhabilité en Afrique. Réinventer les politiques publiques à l'ère néolibérale, Paris, Karthala : 19-45.

LAVIGNE DELVILLE P., 2016, "Pour une socio-anthropologie de l'action publique dans les pays 'sous régime d'aide' ", Anthropologie \& développement, $45: 33-64$.

LECA J., 2012, « L'État entre politics, policies et polity. Ou peut-on sortir du triangle des Bermudes ? ", Gouvernement et action publique, 1(1) : 59-82.

MASSARDIER G., 2003, Politique et action publiques, Paris, Armand Colin. 
OLIVIER DE SARDAN J.-P., 2010, « Développement, modes de gouvernance et normes pratiques (une approche socio-anthropologique) ", Canadian Journal of Development Studies/Revue canadienne d'études du développement, 31(1-2) : 5-20.

OLIVIER DE SARDAN J.-P., 2014, La routine des comportements non-observants au sein des services publics nigériens. Connaître la culture bureaucratique pour la réformer de l'intérieur. Rapport réalisé pour le Haut-Commissariat à la modernisation de l'État, Niamey, LASDEL, $79 \mathrm{p}$.

OLIVIER DE SARDAN J.-P. et PICCOLI E. (éds), 2018, Cash transfers: an anthropological approach. The revenge of contexts, London, Berghahn Press.

OLIVIER DE SARDAN J.-P. et RIDDE V. (éds), 2014, Une politique publique de santé et ses contradictions. La gratuité des soins au Burkina Faso, au Mali et au Niger, Paris, Karthala.

PETITEVILLE F. (éd.), 2017, "La (dé)politisation des organisations internationales ", Critique internationale, 76, dossier, Paris, Presses de sciences po.

RIDDE V. et JACOB J.-P. (éds), 2013, Les indigents et les politiques de santé en Afrique: expériences et enjeux conceptuels, Coll. Investigations d'anthropologie prospective, vol. 6, Bruxelles, Academia.

\title{
Aiviviv
}

\author{
Philippe Lavigne Delville est socio-anthropologue \\ directeur de recherche à l'IRD et président de I'APAD \\ E-mail : philippe.lavignedelville@ird.fr
}

Sylvie Ayimpam est chercheure affiliée à l'Institut des mondes africains

secrétaire générale adjointe de l'APAD

E-mail : ayimpam@mmsh.univ-aix.fr 